\title{
BAKER-AKHIEZER FUNCTION AS ITERATED RESIDUE AND SELBERG-TYPE INTEGRAL
}

\author{
GIOVANNI FELDER \\ Department of Mathematics, ETH Zurich, 8092 Zurich, Switzerland \\ e-mail: giovanni.felder@math.ethz.ch \\ and ALEXANDER P. VESELOV \\ Department of Mathematical Sciences, Loughborough University, Loughborough LE11 3TU, UK \\ and Landau Institute for Theoretical Physics, Moscow, Russia \\ e-mail: A.P.Veselov@lboro.ac.uk
}

\begin{abstract}
A simple integral formula as an iterated residue is presented for the Baker-Akhiezer function related to $A_{n}$-type root system in both the rational and trigonometric cases. We present also a formula for the Baker-Akhiezer function as a Selberg-type integral and generalise it to the deformed $A_{n, 1}$-case. These formulas can be interpreted as new cases of explicit evaluation of Selberg-type integrals.
\end{abstract}

2002 Mathematics Subject Classification. 33E30, 81R12.

1. Introduction. The notion of rational Baker-Akhiezer (BA) function related to a configuration of hyperplanes with multiplicities was introduced in $[4,6,30]$ as a multi-dimensional version of Krichever's axiomatic approach [15]. Such a function exists only for special configurations, in particular for all Coxeter configurations. For the configuration of type $A_{n-1}$ with multiplicity $m$ it has the form

$$
\Psi_{m}^{(n)}=\frac{P_{m}^{(n)}(x, \lambda)}{A(x)^{m} A(\lambda)^{m}} \exp (\lambda, x),
$$

where $A(x)=\prod_{i<j}^{n}\left(x_{i}-x_{j}\right)$ and $(\lambda, x)=\sum_{i=1}^{n} \lambda_{i} x_{i}$, and $P_{m}^{(n)}(x, \lambda)$ is a polynomial in both $x=\left(x_{1}, \ldots, x_{n}\right) \in \mathbb{C}^{n}$ and $\lambda=\left(\lambda_{1}, \ldots, \lambda_{n}\right) \in \mathbb{C}^{n}$ with the leading term $A(x)^{m} A(\lambda)^{m}$. It satisfies the Schrödinger equation

$$
L_{m}^{(n)} \Psi_{m}^{(n)}=-(\lambda, \lambda) \Psi_{m}^{(n)}
$$

where $L_{m}$ is the $n$ particle Calogero-Moser operator

$$
L_{m}^{(n)}=-\Delta+\sum_{i<j}^{n} \frac{2 m(m+1)}{\left(x_{i}-x_{j}\right)^{2}} .
$$

The rational BA function $\Psi_{m}^{(n)}$ is determined uniquely by these properties and has a remarkable symmetry:

$$
\Psi_{m}^{(n)}(z, \lambda)=\Psi_{m}^{(n)}(\lambda, z)
$$


(see [4]). It plays an important role in the theories of commutative rings of differential operators, Huygens principle and quasi-invariants of Coxeter groups [4, 6, 7, 9].

Three ways for computing this function are known. The first one due to Chalykh and one of the authors [6] is known only in the first non-trivial case $m=1$ and is a recursive formula in the number $n$ of variables. The second one uses the iteration of the shift operator (increasing $m$ by one) by Heckman and Opdam, which can be effectively described using the Dunkl operators [11]. The third one, based on the formula of Berest [2], is the most general: it works for all locus configurations (see [4]).

In this paper we present a new formula for the BA function both in rational and trigonometric cases as a simple iterated residue. The structure of the integrand is the same as in other integral formulas known in the theory of Calogero-Moser system and related Jack polynomials (see $[\mathbf{1}, \mathbf{1 3}, \mathbf{1 7}, \mathbf{2 0}]$ ), but the integration cycle is different and adapted only for the case of integer multiplicities.

We present also another formula for the BA function as a Selberg-type integral, which can be considered as the analytic continuation of the residue formula from $m$ to $-m-1$, which is an obvious symmetry of the Calogero-Moser operator. The comparison of these two formulas with other known forms of the BA function can be interpreted as new explicit evaluation of the special Selberg-type integrals, which are probably new. We present similar results also in the deformed $A(n, 1)$ case discovered in $[5,29]$.

Our approach is based on a generalisation of the identity, which plays an important role in the theory of Jack polynomials $[\mathbf{1 9}, \mathbf{2 7}]$ and various versions used in $[\mathbf{1 , 1 3}, \mathbf{1 8}, \mathbf{2 2}]$. In particular, Langmann [18] suggested a simple explanation of this identity within the theory of Calogero-Moser models with different types of particles [16, 23], which is very convenient for us and allows to extend it for the general $A(n, m)$ deformation [25].

2. Rational BA function. The following result can be considered a version of the 'adding particle' approach from [6]. Let us introduce for any two set of variables $u_{1}, \ldots, u_{k}$ and $v_{1}, \ldots, v_{l}$ the function

$$
A(u, v)=\prod_{i=1}^{k} \prod_{j=1}^{l}\left(u_{i}-v_{j}\right)
$$

We will also use the notation

$$
\bar{u}=u_{1}+\cdots+u_{k} .
$$

For fixed distinct $x_{1}, \ldots, x_{k+1} \in \mathbb{C}$ let us choose the cycle of integration $\sigma$ in $k$ integration variables $z_{i}$ as a product of small circles $\left|z_{i}-x_{i}\right|=\epsilon$ around the first $k$ points $x_{i}, i=1, \ldots, k$ and denote by $d z$ the differential form

$$
d z=d z_{1} \wedge d z_{2} \wedge \cdots \wedge d z_{k}
$$

THEOREM 2.1. The BA functions with $k$ and $k+1$ particles are related by the following iterated-residue formula:

$$
\oint_{\sigma} \frac{A(x)^{m+1} A(z)^{m+1}}{A(z, x)^{m+1}} e^{\lambda_{k+1}(\bar{x}-\bar{z})} \Psi_{m}^{(k)}\left(z, \lambda_{1}, \ldots, \lambda_{k}\right) d z=C_{1} \Psi_{m}^{(k+1)}\left(x, \lambda_{1}, \ldots, \lambda_{k+1}\right),
$$


where

$$
C_{1}=C_{1}(k, m, \lambda)=\frac{(2 \pi i)^{k}}{(m !)^{k}} \prod_{i=1}^{k}\left(\lambda_{i}-\lambda_{k+1}\right)^{m}
$$

Iterating this procedure we come to the following formula for the BA function. Note that for $n=1$ the Calogero-Moser operator (3) becomes simply the second derivative and

$$
\Psi_{m}^{(1)}(x, \lambda)=e^{\lambda_{1} x_{1}}
$$

By adding one integration variable at each step we will have $n(n-1) / 2$ integration variables, which we denote $t_{i, j}$ with $1 \leq i \leq j \leq n-1$. It is convenient also to denote $t_{i, n}=x_{i}, i=1, \ldots, n$. The integrand has the following form (cf. $\left.[1,13]\right)$ :

$$
\omega_{m}=\prod_{i \leq j, l \leq j+1 \leq n}\left(t_{i, j}-t_{l, j+1}\right)^{-m-1} \prod_{1 \leq i<l \leq j<n}\left(t_{i, j}-t_{l, j}\right)^{2+2 m} \prod_{l \leq j<n} e^{\left(\lambda_{j}-\lambda_{j+1}\right) t_{l, j}} d t_{l, j} .
$$

We assume that $x_{i}$ are distinct and choose the cycle of integration $\Sigma$ as the product of circles $\left|t_{k, j}-x_{k}\right|=\epsilon(n-j)$ with $\epsilon$ small enough.

COROLLARY 2.2. For any given positive integer $m$ the rational $B A$ function (1) can be given by the following iterated-residue formula:

$$
\Psi_{m}^{(n)}(x, \lambda)=\left(\frac{m !}{2 \pi i}\right)^{\frac{n(n-1)}{2}} e^{\lambda_{n} \bar{x}} A(x)^{1+m} A(\lambda)^{-m} \oint_{\Sigma} \omega_{m} .
$$

To explain another integral representation of BA function note that the CalogeroMoser operator (3) is invariant under the change

$$
m \rightarrow-1-m
$$

This leads to the following formula for BA function as a Selberg-type integral [28].

Let us assume for convenience that $x_{i}, i=1, \ldots, k+1$ have distinct imaginary parts and $\lambda_{i}-\lambda_{j}$ have negative real parts for all $i<j, i, j=1, \ldots, k+1$. Choose the contour of integration $\gamma$ such that $z_{i}=x_{i}+\tau_{i}, i=1, \ldots, k$ with real variables $\tau_{i}$, changing from 0 to $\infty$.

THEOREM 2.3. The rational BA functions for $k$ and $k+1$ particles are related by the following Selberg-type integral formula:

$$
\int_{\gamma} \frac{A(z, x)^{m}}{A(x)^{m} A(z)^{m}} e^{\lambda_{k+1}(\bar{x}-\bar{z})} \Psi_{m}^{(k)}\left(z, \lambda_{1}, \ldots, \lambda_{k}\right) d z=C_{2} \Psi_{m}^{(k+1)}\left(x, \lambda_{1}, \ldots, \lambda_{k+1}\right),
$$

where

$$
C_{2}=C_{2}(m, k, \lambda)=(m !)^{k} \prod_{i=1}^{k}\left(\lambda_{k+1}-\lambda_{i}\right)^{-m-1} .
$$



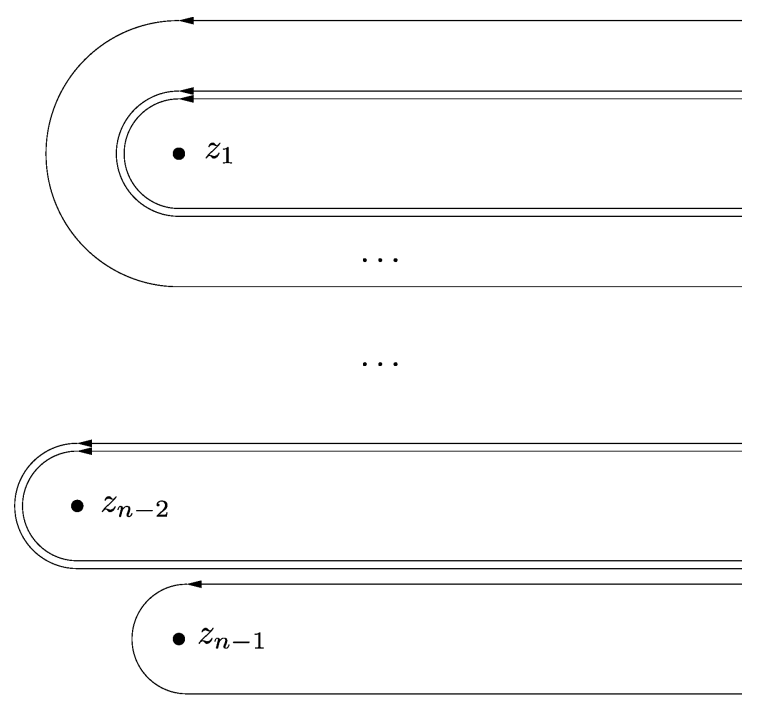

- $z_{n}$

Figure 1. The Pochhammer contour $\Pi$ in the rational case.

Consider now in the same variables $t_{i, j}$ the form $\alpha_{m}=\omega_{-m-1}$,

$$
\alpha_{m}=\prod_{i \leq j, l \leq j+1 \leq n}\left(t_{i, j}-t_{l, j+1}\right)^{m} \prod_{1 \leq i<l \leq j<n}\left(t_{i, j}-t_{l, j}\right)^{-2 m} \prod_{l \leq j<n} e^{\left(\lambda_{j}-\lambda_{j+1}\right) t_{l, j}} d t_{l, j},
$$

and choose the integration contour $\Gamma$ by assuming that $t_{i, j}=t_{i, j+1}+\tau_{i, j}$ with real variables $\tau_{i, j}, 1 \leq i \leq j=1, \ldots, n-1$ changing from zero to infinity.

COROLlARY 2.4. For any positive integer $m$ the rational BA function (1) can be given by the following Selberg-type integral:

$$
\Psi_{m}^{(n)}(x, \lambda)=\left((-1)^{m+1} m !\right)^{-\frac{n(n-1)}{2}} e^{\lambda_{n} \bar{x}} A(x)^{-m} A(\lambda)^{m+1} \int_{\Gamma} \alpha_{m} .
$$

These two different formulas are actually related by analytic continuation. To see this consider the same integral (8) but over Pochhammer contour П:

$$
I_{P}(m)=\int_{\Pi} \alpha_{m}
$$

(see Figure 1). It converges for all $m \in \mathbb{C}$ and is related for positive real $m$ to the Selberg-type integral

$$
I(m)=\int_{\Gamma} \alpha_{m}
$$

in a simple way:

$$
I_{P}(m)=\left(e^{2 \pi i m}-1\right)^{\frac{n(n-1)}{2}} I(m) .
$$


Now replace $m$ ! in (8) by $\Gamma(m+1)$, where $\Gamma(x)$ is the classical Euler gamma function, and note that

$$
\Gamma(m+1)\left(e^{2 \pi i m}-1\right)=2 i e^{\pi i m} \sin \pi m \Gamma(m+1)=(-1)^{m+1} 2 \pi i \Gamma^{-1}(-m)
$$

because of the reflection property of gamma function:

$$
\Gamma(x) \Gamma(1-x)=\frac{\pi}{\sin \pi x} .
$$

We note here a remarkable similarity to Riemann's first proof [21] of the reflection property of the Riemann zeta function

$$
\pi^{-\frac{s}{2}} \Gamma\left(\frac{s}{2}\right) \zeta(s)=\pi^{-\frac{1-s}{2}} \Gamma\left(\frac{1-s}{2}\right) \zeta(1-s) .
$$

3. Trigonometric case. It is actually more convenient for us to use the hyperbolic rather than trigonometric functions, but all the results are automatically applied to both cases because of the algebraic nature of BA function.

The trigonometric version of the $\mathrm{BA}$ function satisfies the equation

$$
\mathcal{L}_{m}^{(n)} \Phi_{m}^{(n)}=-4(v, v) \Phi_{m}^{(n)},
$$

where

$$
\mathcal{L}_{m}^{(n)}=-\Delta+\sum_{i<j}^{n} \frac{2 m(m+1)}{\sinh ^{2}\left(x_{i}-x_{j}\right)}
$$

is the Sutherland operator. It has the form

$$
\Phi_{m}^{(n)}=\frac{P(x, v)}{B(x)^{m} C_{m}(v)} \exp 2(v, x),
$$

where $P(x, v)$ is a trigonometric polynomial in $x$ and a usual polynomial in $v$ with the leading term $B(x)^{m} A(v)^{m}$, where

$$
B(x)=\prod_{i<j}^{n} \sinh \left(x_{i}-x_{j}\right)
$$

and

$$
C_{m}(v)=\prod_{k=1}^{m} \prod_{i<j}^{n}\left(v_{i}-v_{j}-k\right) .
$$

The normalisation constant $C_{m}(v)$ is chosen in such a way that

$$
\lim _{x \rightarrow+\infty} \frac{P(x, v)}{B(x)^{m} C_{m}(v)}=1
$$

when $x \rightarrow+\infty$ in the Weyl chamber $x_{1}>x_{2}>\cdots>x_{n}($ see $[3,8])$. In the exponential variables

$$
u_{i}=\exp 2 x_{i}, i=1, \ldots, n
$$


the $\mathrm{BA}$ function can be rewritten as

$$
\Phi_{m}^{(n)}=\frac{Q(u, v)}{A(u)^{m} C_{m}(v)} u^{v}
$$

where $u^{v}=u_{1}^{v_{1}} \ldots u_{n}^{v_{n}}$ and $Q(u, v)$ is polynomial in $v$ with the leading term $A(u)^{m} A(v)^{m}$.

It is convenient to modify the definition of $A(w, u)$ to include some sign factor as follows:

$$
A^{*}(w, u)=\prod_{i \leq j}\left(w_{i}-u_{j}\right) \prod_{i>j}\left(u_{j}-w_{i}\right)
$$

Let $w=\left(w_{1}, \ldots, w_{k}\right), u=\left(u_{1}, \ldots, u_{k+1}\right)$ and the cycle $\sigma$ similarly to the rational case be the product of circles $\left|u_{i}-w_{i}\right|=\epsilon, i=1, \ldots, k$ with small positive $\epsilon$.

THEOREM 3.1. The trigonometric BA functions $\Phi_{m}^{(k)}\left(w, v_{1}, \ldots, v_{k}\right)$ and $\Phi_{m}^{(k+1)}\left(u, v_{1}, \ldots, v_{k+1}\right)$ are related by the following iterated-residue formula:

$$
\begin{aligned}
& \prod_{i=1}^{k+1} u_{i}^{v_{k+1}} \int_{\sigma} \frac{A(u)^{m+1} A(w)^{m+1}}{A^{*}(w, u)^{m+1}} \\
& \quad \times \prod_{i=1}^{k} w_{i}^{m-v_{k+1}} \Phi_{m}^{(k)}\left(w, v_{1}, \ldots, v_{k}\right) d w=C \Phi_{m}^{(k+1)}\left(u, v_{1}, \ldots, v_{k+1}\right)
\end{aligned}
$$

where

$$
C=(2 \pi i)^{k} \prod_{i=1}^{k}\left(\begin{array}{c}
v_{i}-v_{k+1}-1 \\
m
\end{array}\right)
$$

and

$$
\left(\begin{array}{l}
a \\
m
\end{array}\right)=\frac{a(a-1) \ldots(a-m+1)}{m !} .
$$

As before we introduce $n(n-1) / 2$ integration variables $t_{i, j}$ with $1 \leq i<j \leq n$ with the convention that $t_{i, n}=u_{i}, i=1, \ldots, n$. The integrand in the trigonometric case has the following form (cf. [13]):

$$
\omega_{m}^{*}=\prod_{j=1}^{n-1} A_{j, j+1}^{-m-1} \prod_{j=1}^{n} A_{j, j}^{2+2 m} \prod_{l<j} \prod_{i \leq j}^{n-1} t_{i, j}^{v_{j}-v_{j+1}+m} d t_{i, j},
$$

where

$$
A_{j, j+1}(t)=\prod_{i \leq j, l \leq j+1, i \leq l}^{n}\left(t_{i, j}-t_{l, j+1}\right) \prod_{i \leq j, l \leq j+1, i>l}^{n}\left(t_{i, j}-t_{l, j+1}\right)
$$

and

$$
A_{j, j}(t)=\prod_{1 \leq i<l \leq j \leq n-1}\left(t_{i, j}-t_{l, j}\right)
$$


The cycle of integration $\Sigma$ as before is the product of circles $\left|t_{i, j}-u_{i}\right|=\epsilon(n-j)$ with $\epsilon$ small enough. Note that in contrast to [13] the origin is outside of these circles, so we have no problems with the multi-valuedness of the integrand.

COROLlary 3.2. The trigonometric BA function (12) can be given as an iterated residue

$$
\Phi_{m}^{(n)}(u, v)=C(n, m, v) \prod_{i=1}^{n} u_{i}^{v_{n}} A(u)^{1+m} \int_{\Sigma} \omega_{m}^{*}
$$

with

$$
C(n, m, v)^{-1}=(2 \pi i)^{\frac{n(n-1)}{2}} \prod_{i<j}^{n}\left(\begin{array}{c}
v_{i}-v_{j}-1 \\
m
\end{array}\right)
$$

Similarly to the rational case we have also the following Selberg-type representation of BA functions.

Assume for convenience that the complex numbers $u_{1}, u_{2}, \ldots, u_{k+1}$ have different arguments, and consider the contour $\gamma^{*}$ when $w_{i}$ belongs to the segment joining 0 and $u_{i}$ for $i=1, \ldots, k$. In other words, we assume that $w_{i}=\tau_{i} u_{i}, i=1, \ldots k$ with real $\tau_{i}$ between 0 and 1 . We assume also that $v_{i}-v_{k+1}$ have large positive real parts to guarantee the convergence of the following integral.

THEOREM 3.3. The BA functions $\Phi_{m}^{(k)}$ and $\Phi_{m}^{(k+1)}$ are related by the Selberg-type integral formula

$$
\begin{aligned}
& \prod_{i=1}^{k+1} u_{i}^{v_{k+1}} \int_{\gamma^{*}} \frac{A^{*}(w, u)^{m}}{A(u)^{m} A(w)^{m}} \prod_{i=1}^{k} w_{i}^{-m-1-v_{k+1}} \Phi_{m}^{(k)}\left(w, v_{1}, \ldots, v_{k}\right) d w \\
& \quad=C_{3} \Phi_{m}^{(k+1)}\left(u, v_{1}, \ldots, v_{k+1}\right),
\end{aligned}
$$

where

$$
C_{3}=(-1)^{k m} \prod_{i=1}^{k} \frac{\Gamma(m+1) \Gamma\left(v_{i}-v_{k+1}\right)}{\Gamma\left(v_{i}-v_{k+1}+m+1\right)}
$$

Consider

$$
\alpha_{m}^{*}=\omega_{-m-1}^{*}=\prod_{j=1}^{n-1} A_{j, j+1}^{m} \prod_{j=1}^{n} A_{j, j}^{-2 m} \prod_{l<j} \prod_{i \leq j}^{n-1} t_{i, j}^{v_{j}-v_{j+1}-m-1} d t_{i, j},
$$

and choose the contour of integration $\Gamma^{*}$ such that $t_{i, j}=\tau_{i, j} t_{i, j+1}$ with $\tau_{i, j} \in[0,1]$.

COROLlary 3.4. The trigonometric BA function (12) can be given as a Selberg-type integral

$$
\Phi_{m}^{(n)}(u, v)=C_{4} \prod_{i=1}^{n} u_{i}^{v_{n}} A(u)^{1+m} \int_{\Gamma^{*}} \alpha_{m}^{*}
$$




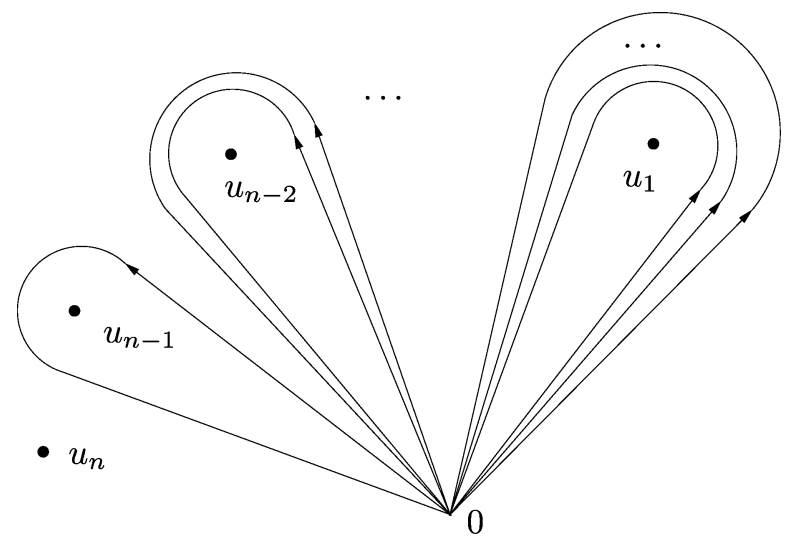

Figure 2. The Pochhammer contour $\Pi$ in the trigonometric case.

with

$$
C_{4}^{-1}=(-1)^{m \frac{n(n-1)}{2}} \prod_{i<j}^{n} \frac{\Gamma(m+1) \Gamma\left(v_{i}-v_{j}\right)}{\Gamma\left(v_{i}-v_{j}+m+1\right)} .
$$

The same calculation as in the rational case shows that these two integral representations are related by analytic continuation. The corresponding analogue of the Pochhammer contour is shown in Figure 2.

4. Deformed case. Consider now the deformed Calogero-Moser operator $[\mathbf{4}, \mathbf{5}]$

$$
L_{m}^{(n, 1)}=-\sum_{i=1}^{n} \frac{\partial^{2}}{\partial x_{i}^{2}}-m \frac{\partial^{2}}{\partial y^{2}}+\sum_{i<j}^{n} \frac{2 m(m+1)}{\left(x_{i}-x_{j}\right)^{2}}+\sum_{i=1}^{n} \frac{2(m+1)}{\left(x_{i}-y\right)^{2}},
$$

corresponding to an additional particle with mass $\frac{1}{m}$ interacting with $n$ CalogeroMoser particles in a special way. According to [5] for any integer $m \in \mathbb{Z}$ it has the eigenfunction (which we will call deformed BA function) of the form

$$
\Psi_{m}^{(n, 1)}=\frac{P(x, y, \lambda, \mu)}{A(x)^{m^{*}} A(x, y) A(\lambda)^{m^{*}} A(\lambda, \mu)} \exp \left((\lambda, x)+\frac{1}{m} \mu y\right),
$$

where $m^{*}=\max (m,-m-1) ; \lambda=\left(\lambda_{1}, \ldots, \lambda_{n}\right) \in \mathbb{C}^{n} ; \mu \in \mathbb{C}$;

$$
A(x)=\prod_{i<j}^{n}\left(x_{i}-x_{j}\right) \text { and } A(x, y)=\prod_{i=1}^{n}\left(x_{i}-y\right)
$$

and $P(x, y, \lambda, \mu)$ is a polynomial in all variables with the highest degree term $A(x)^{m^{*}} A(x, y) A(\lambda)^{m^{*}} A(\lambda, \mu)$.

Let $\Psi_{m}^{(n)}\left(z_{1}, \ldots, z_{n} ; \lambda_{1}, \ldots, \lambda_{n}\right)$ be the BA function (1) from the first section. For positive $m$ we assume as before that all $x_{i}$ have different imaginary parts and that real part of $\lambda_{i}-\mu, i=1, \ldots, n$ are negative and choose the contour $\gamma$ of integration $z_{i}=x_{i}+\tau_{i}$ by considering $\tau_{i} \in \mathbb{R}_{+}, i=1, \ldots, n$. 
For negative $m$ we assume simply that $x_{i}$ are distinct and choose the cycle $\sigma$ as a product of circles $\left|z_{i}-x_{i}\right|=\epsilon, i=1, \ldots, n$.

THEOREM 4.1. The rational deformed BA function (18) for positive integer $m$ can be given by the following Selberg-type integral:

$$
\Psi_{m}^{(n, 1)}(x, y, \lambda, \mu)=\frac{C_{5}}{A(x)^{m} A(x, y)} \int_{\gamma} \frac{A(z, x)^{m} A(z, y)}{A(z)^{m}} e^{\mu\left(\bar{x}-\bar{z}+\frac{y}{m}\right)} \Psi_{m}^{(n)}(z, \lambda) d z
$$

with

$$
C_{5}=\frac{\prod_{i=1}^{n}\left(\mu-\lambda_{i}\right)^{m+1}}{(m !)^{n}} .
$$

For negative $m=-m^{*}-1, m^{*} \in \mathbb{Z}_{+}$it can be represented as an iterated residue

$$
\Psi_{m}^{(n, 1)}=C_{6} \frac{A(x)^{m^{*}+1}}{A(x, y)} \int_{\sigma} \frac{A(z)^{m^{*}+1} A(z, y)}{A(z, x)^{m^{*}+1}} e^{\mu\left(\bar{x}-\bar{z}+\frac{y}{m}\right)} \Psi_{m^{*}}^{(n)}(z, \lambda) d z,
$$

where

$$
C_{6}=\left(\frac{m^{*} !}{2 \pi i}\right)^{n} \prod_{i=1}^{n}\left(\lambda_{i}-\mu\right)^{-m^{*}}
$$

In the trigonometric case (see [5]) we have the operator

$$
\mathcal{L}_{m}^{(n, 1)}=-\sum_{i=1}^{n} \frac{\partial^{2}}{\partial x_{i}^{2}}-m \frac{\partial^{2}}{\partial y^{2}}+\sum_{i<j}^{n} \frac{2 m(m+1)}{\sinh ^{2}\left(x_{i}-x_{j}\right)}+\sum_{i=1}^{n} \frac{2(m+1)}{\sinh ^{2}\left(x_{i}-y\right)},
$$

and the BA function of the form

$$
\Phi_{m}^{(n, 1)}=\frac{Q(u, v, v, \mu)}{A(u)^{m^{*}} A(u, v) C_{m^{*}}(v) C(v, \mu)} u^{v} v^{\frac{\mu}{m}},
$$

where $u_{i}=e^{2 x_{i}}, i=1, \ldots, n ; v=e^{2 y} ; Q(u, v, v, \mu)$ is polynomial in $v \in \mathbb{C}^{n}, \mu \in \mathbb{C}$ with the leading term $A(u)^{m^{*}} A(u, v) A(v)^{m^{*}} A(v, \mu)$; and

$$
C(v, \mu)=\prod_{i=1}^{n}\left(v_{i}-\mu-\frac{1+m}{2}\right)=\prod_{i=1}^{n}\left(v_{i}-\mu+\frac{m^{*}}{2}\right) .
$$

As in the non-deformed case, the form of $C(\nu, \mu)$ is uniquely determined by the property

$$
\lim \frac{Q(u, v, v, \mu)}{A(u)^{m^{*}} A(u, v) C_{m^{*}}(v) C(v, \mu)}=1
$$

when $(u, v) \rightarrow+\infty$ in the chamber $u_{1}>u_{2}>\cdots>u_{n}>v(\operatorname{see}[\mathbf{3}, \mathbf{8}])$.

Let $\Phi_{m}^{(n)}\left(u_{1}, \ldots, u_{n}, v_{1}, \ldots, v_{n}\right)$ be the non-deformed $n$ particle BA function (12). For positive $m$ we assume that all $u_{i}$ have different arguments and that all $\lambda_{i}-v$ are real negative and choose the contour $\gamma^{*}$ of integration $w_{i}=\tau_{i} u_{i}$ with real $\tau_{i} \in[0,1]$. For negative $m=-1-m^{*}$ we assume simply that $u_{i}$ are distinct and choose the cycle $\sigma^{*}$ as a product of circles $\left|w_{i}-u_{i}\right|=\epsilon, i=1, \ldots, n$. 
THEOREM 4.2. The deformed trigonometric BA function $\Phi_{m}^{(n, 1)}(u, v, v, \mu)$ for positive integer $m$ can be given by the Selberg-type integral

$$
\Phi_{m}^{(n, 1)}=C_{7} \frac{\prod_{i=1}^{n} u_{i}^{\mu+\frac{1-m}{2}} v^{\frac{\mu}{m}}}{A(u)^{m} A(u, v)} \int_{\gamma^{*}} \frac{A^{*}(w, u)^{m} A(w, v)}{A(w)^{m}} \prod_{i=1}^{n} w_{i}^{-\mu-\frac{1+m}{2}} \Phi_{m}^{(n)}(w, v) d w
$$

with

$$
C_{7}^{-1}=\prod_{k=1}^{n} \frac{\Gamma(m+1) \Gamma\left(v_{k}-\mu+\frac{1-m}{2}\right)}{\Gamma\left(v_{k}-\mu+\frac{m+3}{2}\right)}
$$

and for negative $m=-m^{*}-1, m^{*} \in \mathbb{Z}_{+}$as an iterated residue

$$
\Phi_{m}^{(n, 1)}=C_{8} \frac{\prod_{i=1}^{n} u_{i}^{\mu+\frac{m^{*}+2}{2}} v^{\frac{\mu}{m}} A(u)^{m^{*}+1}}{A(u, v)} \int_{\gamma^{*}} \frac{A(w)^{m^{*}+1} A(w, v)}{A^{*}(w, u)^{m^{*}+1}} \prod_{i=1}^{n} w_{i}^{-\mu+\frac{m^{*}}{2}} \Phi_{m^{*}}^{(n)}(w, v) d w
$$

where

$$
C_{8}^{-1}=(2 \pi i)^{n} \prod_{i=1}^{n}\left(\begin{array}{c}
v_{i}-\mu+\frac{m^{*}}{2} \\
m^{*}
\end{array}\right)
$$

5. Proofs: main identity. Let

$$
\begin{aligned}
L_{m}^{k, l}(x, y)= & -\left(\frac{\partial^{2}}{\partial x_{1}{ }^{2}}+\cdots+\frac{\partial^{2}}{\partial x_{k}{ }^{2}}\right)-m\left(\frac{\partial^{2}}{\partial y_{1}{ }^{2}}+\cdots+\frac{\partial^{2}}{\partial y_{l}{ }^{2}}\right)+\sum_{i<j}^{k} \frac{2 m(m+1)}{\sinh ^{2}\left(x_{i}-x_{j}\right)} \\
& +\sum_{i<j}^{l} \frac{2\left(m^{-1}+1\right)}{\sinh ^{2}\left(y_{i}-y_{j}\right)}+\sum_{i=1}^{l} \sum_{j=1}^{k} \frac{2(m+1)}{\sinh ^{2}\left(x_{i}-y_{j}\right)}
\end{aligned}
$$

and

$$
\begin{aligned}
L_{m}^{k, l}(x, y)= & -\left(\frac{\partial^{2}}{\partial x_{1}{ }^{2}}+\cdots+\frac{\partial^{2}}{\partial x_{k}{ }^{2}}\right)-m\left(\frac{\partial^{2}}{\partial y_{1}{ }^{2}}+\cdots+\frac{\partial^{2}}{\partial y_{l}{ }^{2}}\right)+\sum_{i<j}^{k} \frac{2 m(m+1)}{\left(x_{i}-x_{j}\right)^{2}} \\
& +\sum_{i<j}^{l} \frac{2\left(m^{-1}+1\right)}{\left(y_{i}-y_{j}\right)^{2}}+\sum_{i=1}^{l} \sum_{j=1}^{k} \frac{2(m+1)}{\left(x_{i}-y_{j}\right)^{2}}
\end{aligned}
$$

be respectively trigonometric and rational deformed Calogero-Moser-Sutherland (CMS) operators in $x, y$ variables [25]. Let $L_{m}^{p, q}$ be a similar operator in variables $z_{1}, \ldots, z_{p}, w_{1}, \ldots, w_{q}$. Let $A(u, v)$ be given by (4),

$$
B(u, v)=\prod_{i=1}^{k} \prod_{j=1}^{l} \sinh \left(u_{i}-v_{j}\right)
$$

and $\bar{x}=x_{1}+\cdots+x_{k}$ as before. 
The key observation ${ }^{1}$ comes from the following result (cf. Langmann [18]).

THEOREM 5.1. The following identity holds for the trigonometric deformed CMS operators:

$$
L_{m}^{k, l} K=L_{m}^{p, q} K+C_{0} K
$$

where

$$
K(x, y ; z, w)=\frac{B(x, z)^{m} B(y, z) B(x, w) B(y, w)^{1 / m}}{B(x)^{m} B(x, y) B(y)^{1 / m} B(z)^{m} B(z, w) B(w)^{1 / m}} e^{\mu\left(\bar{x}-\bar{z}+\frac{1}{m}(\bar{y}-\bar{w})\right)}
$$

and

$$
C_{0}=\frac{1}{4} m^{2}\left[\left(k-p+\frac{1}{m}(l-q)\right)^{3}-\left(k-p+\frac{1}{m^{3}}(l-q)\right)\right]+\mu^{2}\left(p+\frac{q}{m}-k-\frac{l}{m}\right) .
$$

In the rational case we have the same identity (27) for

$$
K(x, y ; z, w)=\frac{A(x, z)^{m} A(y, z) A(x, w) A(y, w)^{1 / m}}{A(x)^{m} A(x, y) A(y)^{1 / m} A(z)^{m} A(z, w) A(w)^{1 / m}} e^{\mu\left(\bar{x}-\bar{z}+\frac{1}{m}(\bar{y}-\bar{w})\right)}
$$

and $C_{0}=\mu^{2}\left(p+\frac{q}{m}-k-\frac{l}{m}\right)$.

The identity (27) with $l=q=0$ goes back to Stanley and Macdonald [19, 27]. In the case $q=0$ and arbitrary $l$ it appeared in [26; see part (iii) in Lemma 3]. To prove it in the general case we borrow the idea from the work of Langmann [18]. It is based on the following result by Sen [23] (see also [16]).

Consider the following generalised CMS operator describing the interacting particles of different masses on a line:

$$
H=-\sum_{j=1}^{N} \frac{1}{m_{j}} \frac{\partial^{2}}{\partial x_{j}^{2}}+\sum_{j<k} \frac{\gamma_{j k}}{\sinh ^{2}\left(x_{j}-x_{k}\right)} .
$$

THEOREM 5.2 (Sen [23]). For the coupling constants of the special form

$$
\gamma_{i j}=\left(m_{i}+m_{j}\right) \beta\left(m_{i} m_{j} \beta-1\right),
$$

where $\beta$ is an arbitrary parameter, the operator (28) has the eigenfunction

$$
\begin{gathered}
\Phi_{0}=\prod_{i<j} \sinh ^{\beta m_{i} m_{j}}\left(x_{i}-x_{j}\right), \\
H \Phi_{0}=E_{0} \Phi_{0},
\end{gathered}
$$

with the eigenvalue

$$
E_{0}=-\frac{\beta^{2}}{3}\left(\left(\sum_{j=1}^{N} m_{j}\right)^{3}-\sum_{j=1}^{N} m_{j}^{3}\right)
$$

\footnotetext{
${ }^{1}$ As we have learnt from Martin Hallnas a similar result can be extracted from his paper with Edwin Langmann [10].
} 
Now we note that $\gamma_{i j}=0$ if $m_{j}=-m_{i}$ or $m_{j}=\left(m_{i} \beta\right)^{-1}$. Choosing

$$
\begin{gathered}
m_{1}=\cdots=m_{k}=1, m_{k+1}=\cdots=m_{k+l}=m^{-1} \\
m_{k+l+1}=\cdots=m_{k+l+p}=-1, m_{k+l+p+1}=\cdots=m_{k+l+p+q}=-m^{-1},
\end{gathered}
$$

where $m=-\beta$, we see that the operator $H$ reduces to the difference

$$
H=L_{m}^{k, l}-L_{m}^{p, q}
$$

of two decoupled deformed CMS operators, and the relation $H \Phi_{0}=E_{0} \Phi_{0}$ implies the identity (27).

We note that this decoupling can be used to characterise the deformed CMS operators among all generalised CMS operators with different masses (28). It does not imply though the quantum integrability, which had to be proven by other means (see $[25,26])$.

The identity (27) suggests that the function $K(x, y, z, w)$ could be used as the kernel of the integral representation transforming the eigenfunctions of one of the deformed CMS operators to another, although to make this precise could be a non-trivial task (see e.g. [22], where a similar problem is discussed).

In particular, choosing in the rational case $k=p+1, l=q=0$ we come to the integral of the type (5). Choosing a suitable cycle (contour) of integration we come to the formula for the BA function. For example, in Theorem 2.1 the cycle $\sigma$ is chosen in such a way that each integration has only one non-zero residue to compute, which guarantees that the result will be of the required form.

The rest of the proof is based on the fact well known in the theory of BA function (see e.g. [8]) that properly normalised trigonometric BA function can be characterised as the special eigenfunction of CMS operator, which is a particular case of the Heckman-Opdam asymptotic solution [12]. The rational case can be treated as the limit of the trigonometric one (see [6]).

6. Examples. In the simplest case $n=2$ the rational BA function is known to have the form (see e.g. [6])

$$
\begin{aligned}
\Psi_{m}^{(2)}= & \left(\lambda_{1}-\lambda_{2}\right)^{-m}\left(D_{12}-\frac{2 m}{x_{1}-x_{2}}\right)\left(D_{12}-\frac{2(m-1)}{x_{1}-x_{2}}\right) \ldots\left(D_{12}-\frac{2}{x_{1}-x_{2}}\right) \\
& \times \exp \left(\lambda_{1} x_{1}+\lambda_{2} x_{2}\right)
\end{aligned}
$$

where

$$
D_{12}=\frac{\partial}{\partial x_{1}}-\frac{\partial}{\partial x_{2}}
$$

We have two different representations for it. The first one is as a residue

$$
\Psi_{m}^{(2)}=\frac{m !\left(x_{1}-x_{2}\right)^{m+1}}{\left(\lambda_{1}-\lambda_{2}\right)^{m}} e^{\lambda_{2}\left(x_{1}+x_{2}\right)} \operatorname{Res}_{z=x_{1}} \frac{e^{\left(\lambda_{1}-\lambda_{2}\right) z}}{\left(z-x_{1}\right)^{m+1}\left(z-x_{2}\right)^{m+1}}
$$


the second one is the integral

$$
\Psi_{m}^{(2)}=\frac{\left(\lambda_{2}-\lambda_{1}\right)^{m+1}}{m !\left(x_{1}-x_{2}\right)^{m}} e^{\lambda_{2}\left(x_{1}+x_{2}\right)} \int_{x_{1}}^{+\infty}\left(z-x_{1}\right)^{m}\left(z-x_{2}\right)^{m} e^{\left(\lambda_{1}-\lambda_{2}\right) z} d z,
$$

which in this case can be effectively computed using the $\Gamma$ integral

$$
\Gamma(a)=\int_{0}^{+\infty} z^{a-1} e^{-z} d z=(a-1) !
$$

for positive integer $a$.

For $n=3$ the corresponding BA function $\Psi_{m}^{(3)}\left(x_{1}, x_{2}, x_{3}, \lambda_{1}, \lambda_{2}, \lambda_{3}\right)$ can be written as follows:

$$
\Psi_{m}^{(3)}=C \operatorname{Res}_{z_{1}=x_{1}} \operatorname{Res}_{z_{2}=x_{2}} \operatorname{Res}_{w=z_{1}} \frac{\left(z_{1}-z_{2}\right)^{2 m+2} e^{\left(\lambda_{2}-\lambda_{3}\right) \bar{z}} e^{\left(\lambda_{1}-\lambda_{2}\right) w}}{\prod_{i=1}^{2}\left(w-z_{i}\right)^{m+1} \prod_{i=1}^{2} \prod_{j=1}^{3}\left(z_{i}-x_{j}\right)^{m+1}},
$$

where

$$
\begin{gathered}
C=\frac{(m !)^{3} \prod_{i<j}^{3}\left(x_{i}-x_{j}\right)^{m+1}}{\prod_{i<j}^{3}\left(\lambda_{i}-\lambda_{j}\right)^{m}} e^{\lambda_{3} \bar{x}} \\
\Psi_{m}^{(3)}=D \int_{x_{1}}^{+\infty} \int_{x_{2}}^{+\infty} \int_{z_{1}}^{+\infty} \\
\times \frac{\prod_{i=1}^{2}\left(w-z_{i}\right)^{m} \prod_{i=1}^{2} \prod_{j=1}^{3}\left(z_{i}-x_{j}\right)^{m} e^{\left(\lambda_{2}-\lambda_{3}\right) \bar{z}} e^{\left(\lambda_{1}-\lambda_{2}\right) w}}{\left(z_{1}-z_{2}\right)^{2 m}} d z_{1} d z_{2} d w
\end{gathered}
$$

with

$$
D=(-1)^{m+1} \frac{\prod_{i<j}^{3}\left(\lambda_{i}-\lambda_{j}\right)^{m+1}}{(m !)^{3} \prod_{i<j}^{3}\left(x_{i}-x_{j}\right)^{m}} e^{\lambda_{3} \bar{x}}
$$

where as before $\bar{x}=x_{1}+x_{2}+x_{3}, \bar{z}=z_{1}+z_{2}$.

One can interpret these formulas either as a new way of representing of BA function or as an explicit evaluation of the Selberg-type integral (36) in terms of the BA function, which can be computed by other methods as well $($ see $[4,6,30])$. The same of course is true for general $n$ and in the deformed case.

We should mention here the work of Kazarnovski-Krol [14], who found explicit expression of certain generalised Selberg integrals using Opdam's results. Our formulas work only for integer values of parameter $m$ but depend on the additional variables $x$.

7. Concluding remarks. It would be interesting to explore the possibilities of choosing different cycles to produce the integral formulas for the super Jack polynomials [26]. For the usual Jack polynomials such integral formulas were obtained in $[\mathbf{1}, \mathbf{1 7}, \mathbf{1 8}, \mathbf{2 0}]$.

Our approach can be also naturally extended to the (deformed) $B C_{n}$ case and related theory of (super) Jacobi polynomials [24]. We will discuss this in more detail elsewhere. 
Acknowledgements. We would like to thank O. Chalykh, M. Hallnas, S. Ruijsenaars, A. N. Sergeev and especially M. Feigin for very useful discussions and comments. APV is grateful to Forschungsinstitut für Mathematik, ETH, Zurich for the hospitality during the spring 2008 when the work has been done. This work has been partially supported by the European Union through the FP6 Marie Curie RTN ENIGMA (Contract number MRTN-CT-2004-5652) and ESF programme MISGAM and by the EPSRC (grant EP/E004008/1).

\section{REFERENCES}

1. H. Awata, Y. Matsuo, S. Odake and J. Shiraishi, Excited states of the CalogeroSutherland model and singular vectors of the $W_{N}$ algebra. Nuclear Phys. B 449(1-2) (1995), 347-374.

2. Y. Berest, Huygens' principle and the bispectral problem, in The bispectral problem (Montreal, PQ, 1997), CRM Proc. Lecture Notes, 14 (American Mathematical Society, Providence, RI, 1998), 11-30.

3. O. A. Chalykh, Bispectrality for the quantum Ruijsenaars model and its integrable deformation. J. Math. Phys. 41(8) (2000), 5139-5167.

4. O. A. Chalykh, M. V. Feigin, and A. P. Veselov, Multidimensional Baker-Akhiezer functions and Huygens' principle. Comm. Math. Phys. 206(3) (1999), 533-566.

5. O. A. Chalykh, M. V. Feigin and A. P. Veselov, New integrable generalizations of Calogero-Moser quantum problem. J. Math. Phys. 39(2) (1998), 695-703.

6. O. A. Chalykh and A. P. Veselov, Commutative rings of partial differential operators and Lie algebras. Comm. Math. Phys. 126(3) (1990), 597-611.

7. P. Etingof and V. Ginzburg, On $m$-quasi-invariants of a Coxeter group. Mosc. Math. J. 2(3) (2002), 555-566.

8. M. Feigin, Bispectrality for deformed Calogero-Moser-Sutherland systems. $J$. Nonlinear Math. Phys. 12(2) (2005), 95-136.

9. M. Feigin and A. P. Veselov, Quasi-invariants of Coxeter groups and $m$-harmonic polynomials. Int. Math. Res. Not. 10 (2002), 521-545.

10. M. Hallnas and E. Langmann, Quantum Calogero-Sutherland type models and generalised classical polynomials. arXiv:math-ph/0703090.

11. G. Heckman, A remark on the Dunkl differential-difference operators, in Harmonic analysis on reductive groups (Brunswick, ME, 1989), Progr. Math., 101 (Birkhäuser Boston, Boston, MA, 1991), 181-191.

12. G. Heckman and E. Opdam, Root systems and hypergeometric functions. I, Comp. Math. 64 (1987), 329-352.

13. A. Kazarnovski-Krol, Cycles for asymptotic solutions and the Weyl group, in The Gelfand Mathematical Seminars, 1993-1995 (Birkhäuser Boston, Boston, MA, 1996), 123-150.

14. A. Kazarnovski-Krol, Variation on a theme of Selberg integral. arXiv:q-alg/9611012.

15. I. M. Krichever, Methods of algebraic geometry in the theory of nonlinear equations. Uspekhi Mat. Nauk. 32(6) (1977), 183-208.

16. V. Y. Krivnov and A. A. Ovchinnikov, An exactly solvable one-dimensional problem with several particle species. Theor. Math. Phys. 50 (1982), 100-103.

17. V. B. Kuznetsov, V. V. Mangazeev and E. K. Sklyanin, Q-operator and factorised separation chain for Jack polynomials. Indag. Mathem., N.S., 14(3,4) (2003), 451-482.

18. E. Langmann, Singular eigenfunctions of Calogero-Sutherland type systems and how to transform them into regular ones. SIGMA 3 (2007), Paper 031, 18.

19. I. G. Macdonald, Symmetric functions and Hall polynomials, 2nd edition (Oxford University Press, New York, 1995).

20. A. Okounkov and G. Olshanski, Shifted Jack polynomials, binomial formula, and applications Math. Res. Letters 4 (1997), 6978.

21. B. Riemann, Ueber die Anzahl der Primzahlen unter einer gegebenen Grösse, Monatsberichte der Berliner Akademie, November 1859, in Gesammelte mathematische Werke, wissenschaftlicher Nachlass und Nachträge (Riemann B., Editor) (Springer-Verlag, Berlin; B. G. Teubner Verlagsgesellschaft, Leipzig, 1990). 
22. S. N. M. Ruijsenaars, Elliptic integrable systems of Calogero-Moser type: Some new results on joint eigenfunctions. In Proceedings of the 2004 Kyoto Workshop on Elliptic integrable systems (Noumi, M. and Takasaki, K., Editors) (Kobe University), 223-240. Available at www.math.kobe-u.ac.jp/publications/rlm18/18elliptic.html.

23. D. Sen, A multispecies Calogero-Sutherland model. Nuclear Phys. B 479(3) (1996), $554-574$.

24. A. N. Sergeev and A. P. Veselov, BC $C_{\infty}$ Calogero-Moser operator and super Jacobi polynomials. arXiv: 0807.3858 .

25. A. N. Sergeev and A. P. Veselov, Deformed quantum Calogero-Moser systems and Lie superalgebras, Comm. Math. Phys. 245 (2004), 249-278.

26. A. N. Sergeev and A. P. Veselov, Generalised discriminants, deformed Calogero-MoserSutherland operators and super-Jack polynomials. Adv. Math. 192(2) (2005), 341-375.

27. R. Stanley, Some combinatorial properties of Jack symmetric functions. Adv. Math. 77(1) (1989), 76-115.

28. A. N. Varchenko, Selberg integrals. arXiv:math/0408308.

29. A. P. Veselov, M. V. Feigin and O. A. Chalykh, New integrable deformations of quantum Calogero - Moser problem. Russian Math. Surveys 51(3) (1996), 185-186.

30. A. P. Veselov, K. L. Styrkas, and O. A. Chalykh Algebraic integrability for Schrödinger equation and finite reflection groups. Theor. Math. Phys. 94(2) (1993), 253-275. 